\title{
Effect of electropolymerization potential on the properties of PEDOT/ZnO thin film composites
}

\begin{abstract}
The physical, chemical and electrochemical properties of poly(3,4-ethylenedioxythiophene) /zinc oxide (PEDOT/ZnO) thin film electrode that were prepared electrochemically were studied. PEDOT was potentiostatically deposited on the hexagonal flake-like ZnO-covered ITO glass substrate by applying three different potentials $(1.0 \mathrm{~V}, 1.25 \mathrm{~V}$ and $1.5 \mathrm{~V})$ where under these circumstances, the effect of varying electropolymerization potentials where studied. The optical bandgap of each of the PEDOT/ZnO composites prepared were noticed to be within the range of $3.40 \mathrm{eV}$ to $3.45 \mathrm{eV}$ which were in between the optical band gap values of sole PEDOT and $\mathrm{ZnO}$. The presence of both materials was asserted from XRD, FTIR and Raman analysis where all of the corresponding peaks for each of the materials in the spectra were assigned. SEM revealed the flake-like hexagonal morphology of $\mathrm{ZnO}$ which is in agreement with the XRD analysis. While the PEDOT morphology was discerned with round-shaped granular morphology where the average grain size was decreased with the electropolymerization potentials. The resistance of charge transfer of PEDOT/ZnO thin film was directly proportional to the electropolymerization potential while on the specific capacitance was inversely proportional. The composite exhibit both PEDOT and ZnO unique properties that can be used as a multi-functional material in various potential applications.
\end{abstract}

Keyword: Electrochemical deposition; Electrochemical impedance spectroscopy; $\mathrm{PEDOT} / \mathrm{ZnO}$ 